\title{
PRODUÇÃO DE BIOETANOL A PARTIR DA HIDRÓLISE ENZIMÁTICA DO BAGAÇO DE CANA-DE-AÇÚCAR
}

\author{
R. F. MOREIRA ${ }^{2}$, O. ALMEIDA ${ }^{1}$, N. T. MACHADO ${ }^{2}$, E. M.S. RODRIGUES ${ }^{1}$, \\ A. M. J. C. NETO ${ }^{3}$, M. A. CORDEIRO ${ }^{2}$, B. R. CHAGAS $^{1}$, E. L. G. TENORO ${ }^{1}$, M. L. F. \\ TEIXEIRA $^{3}$, P. R.VEIGA DA SILVA ${ }^{3}$ \\ ${ }^{1}$ Universidade Federal Do Pará/Instituto de Ciências Exatas e Naturais/Faculdade de Química \\ ${ }^{2}$ Universidade Federal Do Pará/Instituto de Tecnologia/Faculdade de Engenharia Química \\ ${ }^{1}$ Universidade Federal Do Pará/Instituto de Ciências Exatas e Naturais/Faculdade de Física \\ E-mail para contato: rosiane03@ hotmail.com
}

\begin{abstract}
RESUMO -O aproveitamento de recursos renováveis representa uma importante atividade econômica no cenário global de desenvolvimento tecnológico, uma vez que a exploração adequada de recursos naturais com potencial energético, como fonte de energia e de insumos para a indústria é um dos principais fatores de ascensão econômica atual dos países em desenvolvimento. A utilização de enzimas em processos fermentativos de resíduos celulósicos tem conquistado um lugar de destaque neste contexto. $\mathrm{O}$ objetivo principal deste trabalho foi em avaliar a utilização da combinação das enzimas comerciais Xylanase (NS22083), $\beta$-glucanaseXylanase (NS22002) e $\beta$ glucanase (NS22118) na proporção de 0,6:0,3:1,7, em massa,na produção de glicosea partir do bagaço de cana-de-açúcar, após pré-tratamento alcalino com solução de hidróxido de sódio $(6 \% \mathrm{~m} / \mathrm{v})$ em quatro temperaturas, ambiente $\left(28,5^{\circ} \mathrm{C}\right)$, a 70 , a $90{ }^{\circ} \mathrm{C}$ (SPAC, PAC70 e PAC90), durante 24 horas e a $120^{\circ} \mathrm{C}$ (PAC120), durante 1 hora, seguido de hidrólise enzimática para a conversão de celulose em glicose. As fermentações ocorreram a $50 \pm 2{ }^{\circ} \mathrm{C}$. Os rendimentos no processo de hidrólise enzimática foram de 23,87\% (SPAC), 45,85\% (PAC70),51,99\% (PAC90) e 90,85\% (PAC120). Os resultados obtidos sugerem que as taxas de conversão dos resíduos celulósicos em glicose são fortemente dependentes da temperatura no processo de polpação alcalina.
\end{abstract}

\section{INTRODUÇÃO}

O bagaço de cana é usado atualmente como a principal fonte de energia necessária em usinas de açúcar e destilarias de álcool e também para a geração de energia elétrica, etc. (Rochaet. al., 2011). Ele é um material lignocelulósico mais estudado para a produção de etanol de segunda geração sem necessidade de incrementos na área de plantio (Albarelli, 2013). Substâncias lignocelulósicos, tais como palhas de cereais estão disponíveis em grandes quantidades e pode ser facilmente fermentado para produzir o etanol, que pode ser usado tanto como um combustível para motores em forma pura ou como um componente de mistura de gasolina (Singhet. al., 2014).

No Brasil, o resíduo mais abundante é o bagaço de cana de açúcar que é constituído principalmente por celulose, hemicelulose, lignina e pequenas quantidades de produtos extraíveis e 


\section{9 a 22 de outubro de 2014 \\ Florianópolis/SC}

sais minerais (Albarelli, 2013; Dias, 2011; Rocha, et al., 2011). A cana-de-açúcar pertence ao gênero Saccharum, da família das gramíneas e sua composição pode variar em função do tipo de cana, do solo, do clima, da disponibilidade de água e da época na safra, dentre outros aspectos (De Souza, 2006).

Para a produção de etanol é requerida algumas etapas principais como: pré-tratamento da biomassa que podem ser térmicos, químicos, físicos, biológicos ou uma combinação de todos esses, o que dependerá do grau de separação requerido e do fim proposto; hidrólise enzimática da celulose para obtenção de monossacarídeos onde se tem altos rendimentos fermentescíveis sem formação de inibidores e à não-degradação da glicose que é realizada a temperaturas brandas, em torno de $50^{\circ} \mathrm{C} \mathrm{e}$ pH de 4,8 para disponibilizar a celulose ao ataque enzimático; a fermentação e destilação o bagaço seguiria os mesmos processos na produção de etanol convencional (Albarelli, 2013; Dos Santos, 2009 Silva, 2010).

O objetivo deste trabalho foi avaliar a utilização da combinação das enzimas Xylanase (NS22083), $\beta$-glucanaseXylanase (NS22002) e $\beta$-glucanase (NS22118) na proporção de 0,6:0,3:1,7 $(\mathrm{m} / \mathrm{m})$ na produção de glicose a partir do bagaço de cana-de-açúcar, após pré-tratamento alcalino com solução de hidróxido de sódio $(6 \% \mathrm{~m} / \mathrm{v})$ em quatro temperaturas, ambiente $\left(28,5^{\circ} \mathrm{C}\right)$, a 70 , a $90{ }^{\circ} \mathrm{C}$ (SPAC, PAC70 e PAC90)e a $120^{\circ} \mathrm{C}$ (PAC120) no processo de hidrólise enzimática.

\section{MATERIAIS E MÉTODOS}

\subsection{Bagaço de Cana de Açúcar}

As amostras do bagaço de cana de açúcar foram obtidas nas feiras livres de Belém-PA no mês de Outubro de 2013. A preparação do bagaço consistiu de lavagem, seguida de secagem à temperatura ambiente durante $72 \mathrm{~h}$, secagem em estufa com circulação de ar (MARCONI-035) a $50 \pm 2{ }^{\circ} \mathrm{C}$, até atingir peso constante.

O material seco foi triturado em moinho de facas (WILLEY-BT 602) e acondicionado em sacos plásticos. O tamanho médio das partículas do bagaço utilizado foi de 6 a $50 \mathrm{~mm}$ de comprimento e $0,3 \mathrm{~mm}$ de diâmetro de um filamento único sendo $3 \mathrm{~mm}$ o diâmetro do aglomerado. Essas medidas foram realizadas utilizando um paquímetro Mitutoyo (0 a $250 \mathrm{~mm})$.

Os teores de umidade e de cinzas, foram determinadas conformea AssociationofOfficialAnalyticalChemists, métodos $\mathrm{n}^{\circ} 940.26$ (1984) e $\mathrm{n}^{\circ} 964.22$ (2000).O teor de lignina foi determinado pela adaptação do método de Klasson modificado por Rocha et al., 1997.

\subsection{Polpação Alcalina}

O processo de polpação foi realizado em um Erlenmeyer (3L), contendo $150 \mathrm{~g}$ do bagaço de cana-de-açúcar seco e moído, seguido da adição de solução de $\mathrm{NaOH}(2 \mathrm{~L})$ a $6 \%$ (m/v). Foram preparadas quatro polpas do bagaço nas mesmas condições, sendo que a primeira (SPAC), polpação realizada em temperatura ambiente $\left(28,5^{\circ} \mathrm{C}\right.$ ), a segunda PAC70) e a terceira (PAC90) foram submetidas a aquecimento em estufa a 70 e $90{ }^{\circ} \mathrm{C}$, durante 24 horas. A quarta foi auto clavada a 120 ${ }^{\circ} \mathrm{C}$ durante 60 minutos.

Após este período, todas as soluções foram filtradas e os resíduos, lavados com água corrente 


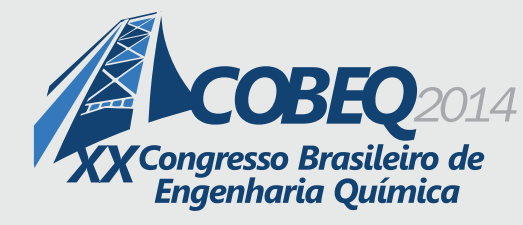

\section{9 a 22 de outubro de 2014 \\ Florianópolis/SC}

e neutralizados com ácido sulfúrico 1:8 (v/v), posteriormente, os resíduos foram submetidos a secagem em estufa a $105{ }^{\circ} \mathrm{C}$ até peso constante.Os rendimentos são expressos pela razão entre a massa de resíduo seco em relação à massa da amostra seca.

\subsection{Atividade e Hidrólise Enzimática}

As enzimas comerciais empregadas neste trabalho corresponderam às preparações Xylanase (NS22083), $\beta$-glucanaseXylanase (NS22002) e $\beta$-glucanase (NS22118) (Novozymes, Bagsvaerd, Denmark), cedidas pela NovozymesLatin América (Araucária, PR).

Para a determinação da atividade celulósica total das enzimas, utilizou-se o método de açúcares redutores (AR), baseada na metodologia proposta por Ghose (1987). Para os ensaios de hidrólise tomou-se como base as relações do complexo enzimático em massa de 0,6:0,3:1,7 (xylanase: $\beta$-glucanasexylanase: $\beta$-glucanase).Foram utilizados $10 \mathrm{~g}$ do bagaço pré-tratados (SPAC, PAC70, PAC90 e PAC120) como fonte de carbono. Os ensaios foram conduzidos em Erlenmeyer de $500 \mathrm{~mL}$ contendo $200 \mathrm{~mL}$ de água destilada. $\mathrm{O} \mathrm{pH}$ foi ajustado para 4,8, em seguida, foi adicionado a mistura do complexo enzimático na relação 1:2,6 (substrato:enzima).

A incubação foi realizada em estufa microbiológica a $50 \pm 3{ }^{\circ} \mathrm{C}$ com agitação periódica. Após o tempo de incubação (7 dias) foi realizada a inativação enzimática com o aumento da temperatura para $90{ }^{\circ} \mathrm{C}$ por 1 hora.As concentrações de açúcares redutores foram determinadas pelo método do ácido 3,5-dinitrosalicílico (DNS) descrito por Miller (1959), mediante a construção da curva padrão de glicose nas concentrações de 0,1 a 1,0 g.L ${ }^{-1}$. A concentração de glicose foi determinada em um espectrofotômetro digital (BIOSPECTRO, Faixa de 340-1000 $\mathrm{nm}$, Modelo SP-12) a $540 \mathrm{~nm}$.

\section{RESULTADOS E DISCUSSÃO}

Os teores de umidade e o rendimento do bagaço em base seca foram 88,37\% e 11,63\%, respectivamente. Os teores de cinzas para o bagaço de cana-de-açúcar sem pré-tratamento (SPAC) e nos resíduos obtidos após a polpação alcalina (PAC70, PAC90 e PAC120), foram de 2,05 \pm 0,027, $0,62 \pm 0,013 \%, 0,48 \pm 0,007$ e $0,18 \pm 0,003$. Os altos teores de lignina no bagaço de cana sem prétratamento (SPAC) $(20,08 \pm 0,006 \%)$ e do resíduo pré-tratado PAC70 (13,03 \pm 0,009\%), mostraramse menos susceptíveis ao pré-tratamento, e consequentemente, baixa conversão por vias fermentativas.

Comparativamente ao bagaço de cana, os baixos teores de cinzas presentes nos resíduos (PAC70, PAC90 e PAC120) favorecem o potencial energético no processo de fermentação, e não acarreta um aumento na capacidade tamponante da matéria-prima, levando a uma neutralização parcial dos catalisadores ácidos responsáveis pela hidrólise parcial dos polissacarídeos constituintes da biomassa.

A determinação do tempo ideal de fermentação e de incubação usando os resíduos do bagaço de cana de açúcar pré-tratados (PAC70, PAC90 e PAC120) foi realizado mediante a avaliação da atividade enzimática (Figura 1).

Os resultados mais expressivos foram obtidos em um tempo de $120 \mathrm{~h}$, foram de 171,89 (SPAC), 330,13 (PAC70) e 140,37 (PAC90) e 245,30 (PAC120) U.mL ${ }^{-1}$, respectivamente. 


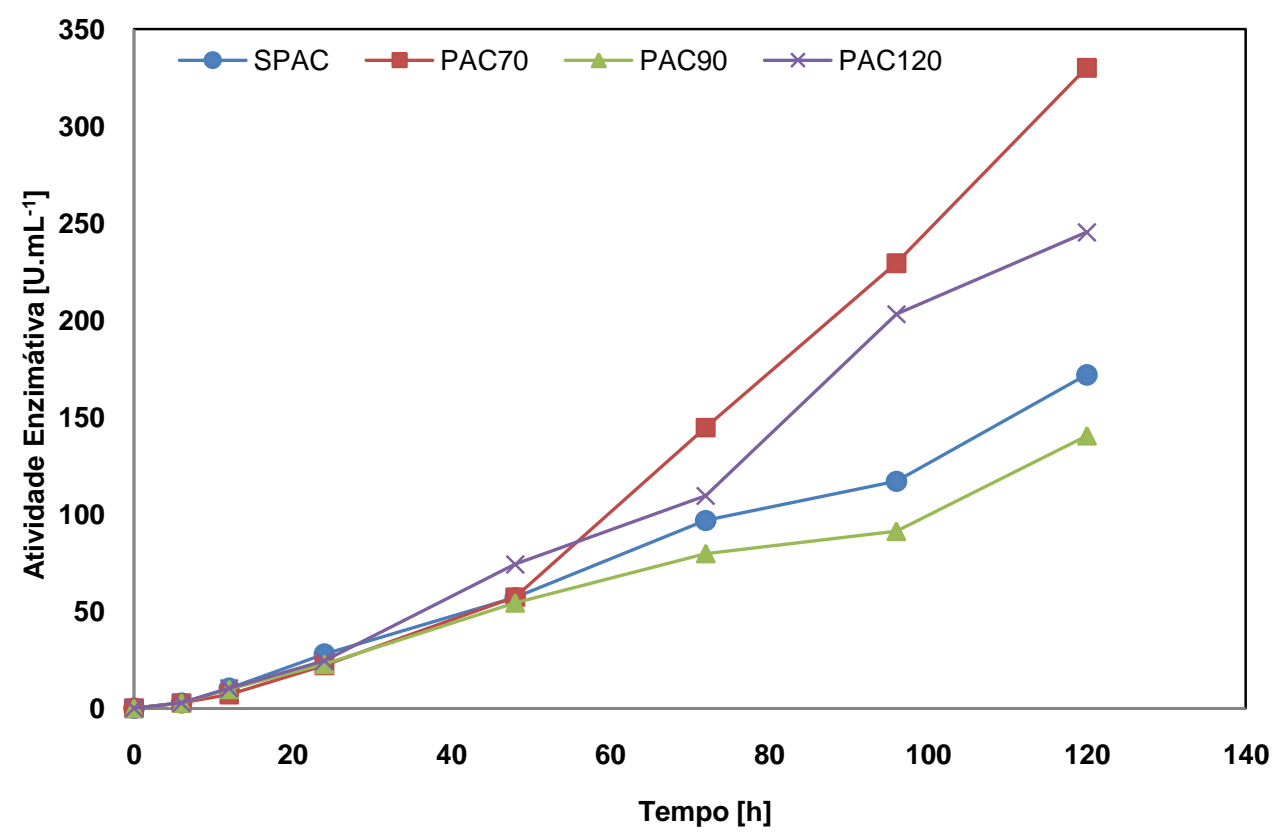

Figura 1. Atividade enzimática.

Os altos teores de lignina no bagaço de cana sem pré-tratamento (SPAC) $(20,86 \pm 0,006 \%)$ e do resíduo pré-tratado PAC70 $(13,03 \pm 0,009 \%)$, mostraram-se menos susceptíveis ao pré-tratamento e consequentemente baixa conversão por vias fermentativas.

A eficiência com que a celulose é hidrolisada depende de muitos fatores que envolvem desde as características do substrato até a natureza do sistema enzimático utilizado. Os fatores relacionados às enzimas incluem a inibição do complexo celulásico pelo acúmulo de produto final (glucose e celobiose), adsorção irreversível das enzimas sobre o substrato, desnaturação enzimática por exposição excessiva à temperatura e agitação necessárias ao processo. Já os fatores relacionados ao substrato correspondem ao teor de lignina, porosidade, cristalinidade das fibras de celulose e o teor de hemiceluloses.

Em paralelo aos resultados de atividade enzimática foram determinados os teores de açúcares redutores (AR) para os resíduos do bagaço de cana de açúcar pré-tratados. Os resultados obtidos indicam uma variação dos teores de AR's, no final do processo de hidrólise enzimático, os teores médios de AR's para os resíduos pré-tratados (SPAC, PAC70, PAC90 e PAC120) foram de 23,87, $45,85,51,99$ e 90,85\%, respectivamente, conforme Figura 2. 


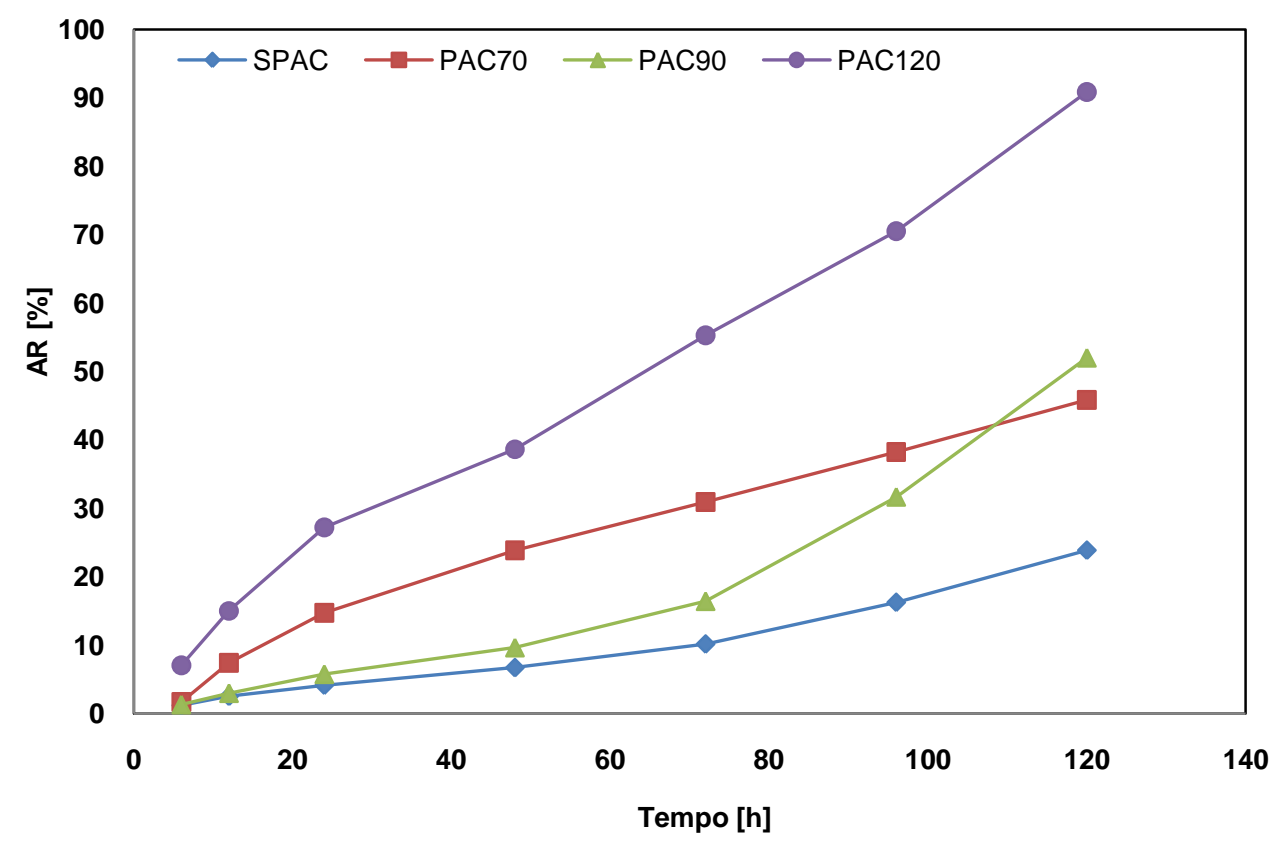

Figura 1. Teor de açúcares redutores no processo de hidrólise enzimática do bagaço de cana-deaçúcar.

\section{CONCLUSÃO}

O bagaço de cana-de-açúcar submetido a caracterização via umidade, cinzas, ART, polpação alcalina, lignina, atividade enzimática e fermentação alcoólica apresentou os melhores resultados quando submetido a pré-tratamento a $120^{\circ} \mathrm{C}$.

Nesta temperatura o substrato insolúvel PAC120 apresentou teores de lignina de 0,18 $\pm 0,003 \%$, consequentemente apresentando a melhor taxa de conversão de resíduos celulósico em glicose $(90,85 \%)$ e rendimento de $85,35 \%$ no processo de fermentação alcoólica.

Os resultados obtidos permitem afirmar que as taxas de conversão dos resíduos celulósicos em glicose são fortemente dependentes da temperatura, quando utilizada a proporção de substrato-enzima de 0,6:0,3:1,7, respectivamente para a mistura do complexo enzimático Xylanase (NS22083), $\beta$ glucanaseXylanase (NS22002) e $\beta$-glucanase (NS22118).

\section{REFERÊNCIAS}


Albarelli, J. Q. Produção de Açúcar e Etanol de Primeira e Segunda Geração: Simulação, Integração Energética e Análise Econômica- Tese de Doutorado - Campinas, 2013.

AOAC. ASSOCIATION OF OFFICIAL ANALYTICAL CHEMISTS. Official methods of analysis. Edited by Patricia Cunniff .17th ed., v.2., cap.37, 42 e 44, 2000.

AOAC. ASSOCIATION OF OFICIAL ANALYTICAL CHEMISTS. Official methods of analysis. 14th ed. Arlingyon: Sidney Willians, 1984.

DE ANDRADE, R. R. Modelagem Cinética do Processo de Produção de Etanol a Partir de Hidrolisado Enzimático de Bagaço de Cana-de-açúcar Concentrado com Melaço Considerando Reciclo de Células - Tese de Doutorado- Campinas, 2012.

DE SOUZA, C. S. Derivatização Química e Caracterização de uma Lignina do Bagaço da Cana-de-açúcar - Dissertação - Uberlândia-MG, 2006.

DIAS, M. O. S. Desenvolvimento e Otimização de Processos de Produção de Etanol de Primeira e Segunda Geração e Eletricidade a partir da Cana-de-açúcar - Tese de Doutorado- Campinas, 2011.

DOS SANTOS, J. R. A., GOUVEIA, E. R. Produção de Bioetanol de Bagaço de Cana-deaçúcar- Revista Brasileira de Produtos Agroindustriais, Campina Grande, v.11, n.1, p.27-33, ISSN 1517-8595, 2009.

GHOSE, T. K. International Union of Pure and Applied Chemistry- Measurement ofCellulase Activities. v.59, n.2, p.257-268, 1987.

MALDONADE, I. R., CARVAHO, P. G. B., FERREIRA, N. A. Protocolo para a Determinação de Açucares Totais em Hortaliças pelo Método de DNS - ISSN 1414.9850, Março, 2013.

ROCHA, G. J. M., MARTIN, C., SOARES, I. B., MAIOR, A. M. S., BAUDEL, H. M., DE ABREU, C. A. M. Dilutemixed-acid pretreatment of sugarcane bagasse for ethanol production- Biomass and Bioenergy, v.35, p.663- 670, 2011.

ROCHA, G. J. M.; SILVA, F. T.; CURVElO, A. A. S; ARAÚJO, G. T.; Resumos do 5th Brazilian Symposium on the Chemistry of Lignins and Other Wood Components, Paraná, Brasil, 1997.

SILVA, O. G. Produção de Etanol com a Utilização do Bagaço de Cana-de-açúcarMonografia - Faculdade de Tecnologia de Araçatuba - Curso de Tecnologia de Biocombustíveis Araçatuba, 2010. 
SINGH, R., SHUKLA, A., TIWARI, S., SRIVASTAVA; M. A review on delignification of lignocellulosic biomass for enhancement of ethanol production potential- Renewable and Sustainable Energy Reviews, v.32, p.713-728, 2014. 